\title{
FFHD
}

Functional Foods in Health and Disease

\section{Health benefits of olive oil and by-products and possible innovative applications for industrial processes}

\section{Mariangela Centrone*, Marianna Ranieri*, Annarita Di Mise, Mariagrazia D’Agostino, Maria Venneri, Giovanna Valenti, Grazia Tamma**}

Department of Biosciences, Biotechnologies and Biopharmaceutics, University of Bari, Italy

*These authors contributed equally: Mariangela Centrone and Marianna Ranieri

${ }^{* *}$ Corresponding Author: Grazia Tamma, PhD, Professor, Department of Biosciences, Biotechnologies and Biopharmaceutics, University of Bari, Aldo Moro, Bari, Italy.

Submission Date: April 9th 2021 ; Acceptance Date: June 14 ${ }^{\text {th }}, 2021$; Publication Date: July $16^{\text {th }}, 2021$

Please cite this as: Centrone M., Ranieri M., Di Mise A., D'Agostino M., Venneri M., Valenti G., Tamma G. Health benefits of olive oil and by-products and possible innovative applications for industrial processes. Functional Food in Health and Disease 2021. 11(7): 295-309. DOI: https://www.doi.org/10.31989/ffhd.v11i7.800

\section{ABSTRACT}

In the last few years, the production of olive oil has incredibly increased due to its beneficial properties on human health. On the other hand, the amount of waste and by-products derived from the olive oil industry has increased as well, prompting the development of new fields of research aiming to reduce disposal costs and valorize bioactive compounds from the viewpoint of the green economy. Interestingly, olive oil and by-products contain numerous molecules, including phenols, exhibiting pleiotropic effects in both in vitro and in vivo studies. This review aims to describe the more recent studies focusing to identify and describe the potential roles and the new applications of extracts isolated from wastes and by-products or specific compounds, such as hydroxytyrosol (HT) and oleuropein. Extracts or bioactive molecules from olive oil and by-products have been proposed as a food additive, to produce biofuel, for animal feeding, and to create new matrices in bioremediation protocols. Moreover, bio-compounds have been described to have numerous beneficial effects on human health as they reduce the risk of developing certain chronic diseases. Despite the already published studies, more research is necessary to valorize bioactive compounds in olive oil and by-products, as well as their potential use to improve human health and green applications in different industrial processes.

Keywords: extra virgin olive oil, by-products, olive leaf extract, bioactive compounds, human health, and industrial application 


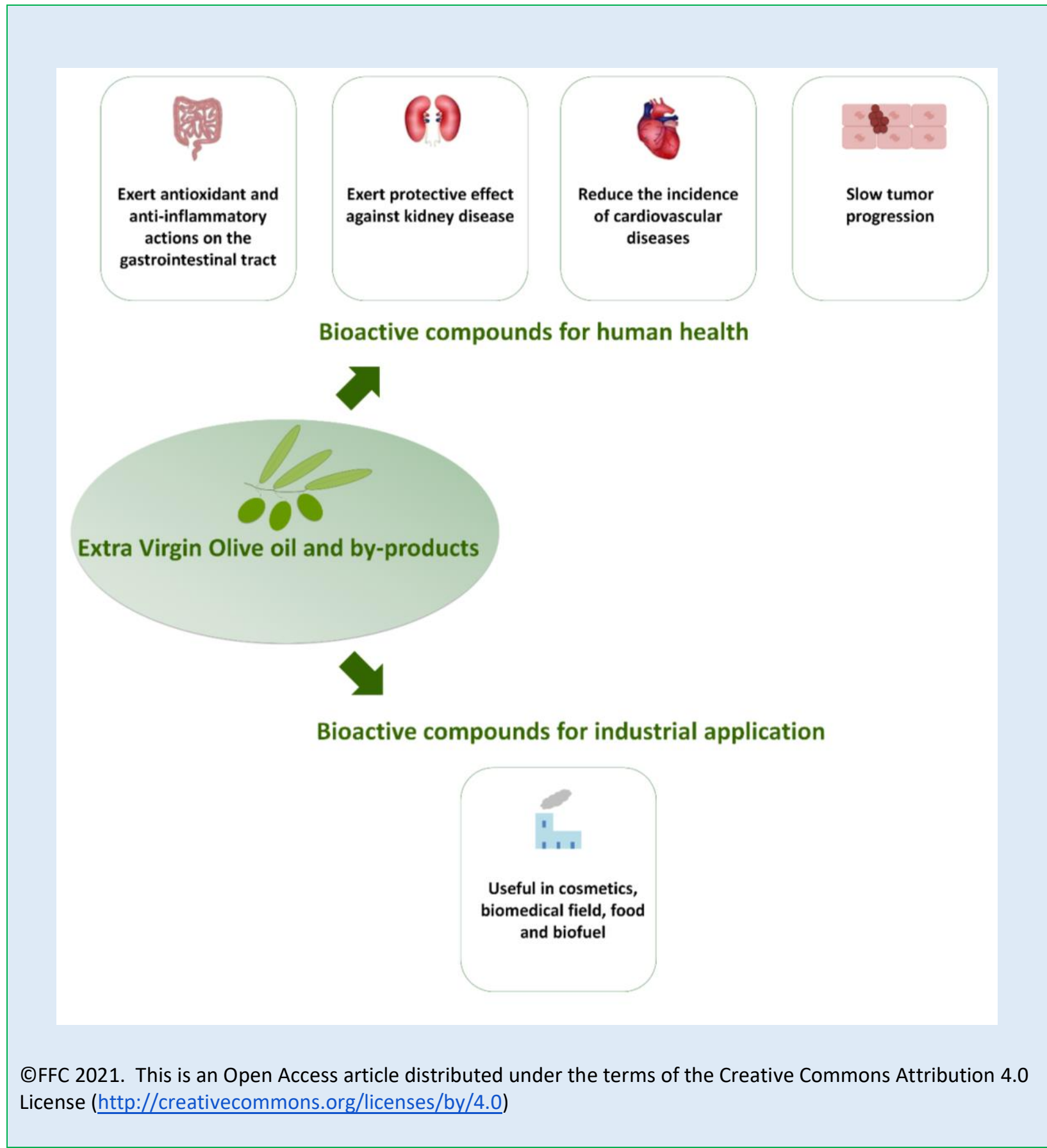

\section{INTRODUCTION}

In the last few years, the consumption of olive oil has increased significantly throughout the world for its organoleptic and healthy features. The increased demand for olive oil has been accompanied by the development of new and green technologies aimed to reduce the disposal cost of wastes and to valorize byproducts as they contain numerous added-value compounds that can have a variety of applications.
Olive leaves have a high content of antioxidant compounds, including phenols (hydroxytyrosol, or tyrosol), and secoiridoids such as oleuropein, flavonoids including, luteolin, hesperetin, and diosmetin. Secoiridoids include several bioactive compounds found expressed in all species of Oleaceae plants. The composition of bioactive molecules in the leaves depends on plant variety and season harvesting [1-6]. In the leaves, oleuropein is 
the most abundant secoiridoid, having welldocumented health effects. Oleuropein displays free radical scavenging and metal-chelating activities that are crucial to contrast membrane lipid oxidation [7].

Olive pomace (OP), and olive wastewater (OWW) contain an important fraction of phenolic compounds [6]. In particular, OWW is enriched with hydroxytyrosol $(\mathrm{HT})$, which is considered a potent antioxidant compound. In this respect, HT-enriched extracts are marketed as food supplements and patented for their organoleptic and healthy properties [4]. The beneficial effects of olive oil byproducts are mainly due to their antioxidant and antiinflammatory potential. Dysregulation in the processes controlling the intracellular level of reactive species (RS) often characterizes several conditions, including aging and frailty. It is now well accepted that a high level of intracellular reactive species can be associated with chronic diseases, thyroid dysfunction, cancer, and several age-related disorders such as atherosclerosis, hypertension, cardiovascular dysfunction, etc. [8]. Emerging evidence revealed that RS can modulate intracellular signaling by functioning as messenger molecules. Hence, the intracellular roles of these molecules have been deeply revised. Membrane receptors and channels might be involved in sensing and transporting RS such as hydrogen peroxide and nitric peroxide [9]. On the other hand, oxidative molecules can also control the functionality of the membrane transport system through oxidative-sensitive post-translational modifications [10]. Indeed, several transporters and channels can be subjected to S-glutathionylation, nitrosylation, or carbonylation that may alter protein stability, localization, and function. Numerous phytocompounds in food have been demonstrated to provide beneficial effects to human health as they may participate and modulate several physiological processes by interfering with signal transduction pathways controlling inflammation, cell growth, and survival. Numerous studies revealed that bioactive molecules in EVOO and olive by-products have hormone-interfering features [11-12]. In euthyroid animals, administration of olive oil or OLE increased the active triiodothyronine (T3) and the inactive thyroxine (T4) and reduced the thyroid-stimulating hormone (TSH) [12-14]. Furthermore, oral administration of EVOO improves the TSH profile and the oxidative status of the thyroid gland in a rat model of hypothyroidism [15]. Nevertheless, the molecular signals responsible for these beneficial effects are still not known.

Leaves and by-products, derived from agricultural processes, contain a high level of bioactive molecules and certain phenols that can be marketable as nutraceuticals or in the pharmaceutical and cosmetic industry [16]. Bioactive compounds in olive by-products could selectively be extracted and applied for different industrial processes. These principles represent the core of the circular economy, a model aiming to valorize by-products and wastes making the industrial processes more sustainable. Wastes and by-products are most valuable when used as pharmaceuticals for human health. This review provides an update on the functional characterization and application of olive leaves and by-products extracts that are enriched with bioactive molecules and several polyphenols displaying antioxidant activity.

Exploitation and alternative use of olive oil byproducts: Olive oil demand is rapidly expanding worldwide due to its nutraceutical properties. Olive oil production is typical of the Mediterranean area, and it is developing in several other regions of the world sharing similar environmental and climate 
conditions. The production process, however, generates a huge amount of waste, mainly pomace (OP) and mill wastewater (OWW), that create serious environmental issues due to the high and toxic concentration level of polyphenols. Moreover, tons of pruning residues are also generated from regular cultivation. This huge amount of biomass is usually used for energy production, composting, and recently also biofuel [17-18]. Notably, the high level of bioactive compounds in the by-products and their recovery offers a great challenge and a unique opportunity for the further development of the olive oil industry in the optical of the circular economy (Figure 1). Therefore, green extraction technologies that include the use of environmentally friendly solvents aiming to preserve the functionality of phytocompounds should always be applied [2]. The use of olive by-products for animal feeding is also well established. Dried olive cake has been successfully supplemented in the diet of different farm animals as it does not alter blood parameters and serum enzyme activities [19-20]. Also, OWW has been supplemented in the sheep diet to produce high-quality cheese [21]. Moreover, the olive extract has been applied to generate edible biofilm displaying antioxidant and antibacterial properties against Escherichia coli and Staphylococcus aureus [22]. Also, extracts from olive oil by-products were incorporated into different food matrixes including sunflower oil, thereby increasing antioxidant potential [23]. Alternative use of olive by-products includes the generation of selective biosorbent surfaces for the removal of environmental pollution induced by heavy metals like $\mathrm{Pb}$ and $\mathrm{Cd}$ [24]. In this respect, we have recently demonstrated that green olive leaf extracts (OLE) impaired cytotoxic effects induced by exposure to a low concentration level of cadmium. In the renal collecting duct MCD4 cells exposed to $\mathrm{Cd}$, treatment with OLE decreased the frequency of micronuclei, DNA double-strand breaks, actin depolymerization and S-glutathionylation, and generation of ROS [5]. Moreover, thyroid deregulation can cause metabolic diseases and affect skeletal development. Treatment with oleuropein and HT showed beneficial actions to protect against bisphenol A-induced hypothyroidism [25]. Interestingly, olive pruning residues and OWW also have been applied as a substrate to grow edible mushrooms. In that study, the residue and OWW reduced certain pollution parameters of olive byproducts, bio-converting them in high-quality biomass as these mushrooms are enriched with glucans and phenols that give health benefits [18]. Lately, it has been shown that oleuropein and HT in extracts obtained from leaves or OWW, respectively, display antiviral activities against the SARS-COV2, possibly by inducing structural changes of the virus [26-27]. Hydrogel or cream enriched with HT has been proposed as an antiviral hand cream [26]. On another hand, it has been proposed that phytosomal formulation containing luteolin (in olive pomace oil) may mitigate some of the symptoms observed in long-COVID patients [28].

By-products, derived from olive oil production, have been used in the cosmetic and food industry for their antioxidant supply. Consistently, bioactive compounds from OWW are now marketable as biopreservatives useful to improve the product's shelf life [23]. Indeed, the addition of OLE to olive oil increased the quality and the shelf life of baked snacks [29-30] due to a reduction of oxidative degradation assessed by the oven test [30]. Also, dried olive paste has been used to prepare ready-tocook cod sticks breaded that were enriched with total phenols and flavonoids compared to control foods that were not treated with olive by-products [31]. 


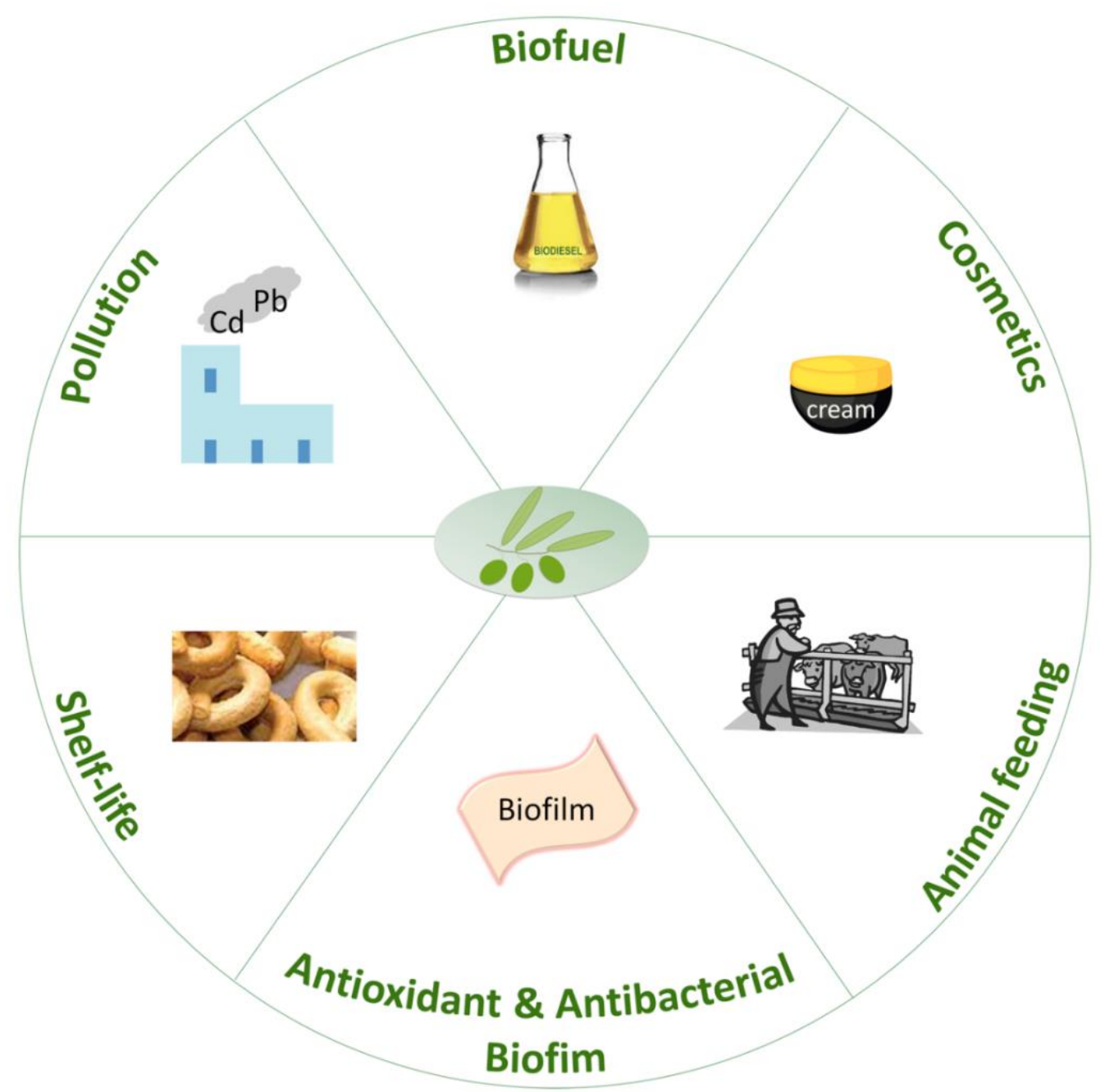

Figure 1. Diagram showing industrial application of olive oil and olive-by-products: as a food additive, to produce biofuel, for animal feeding, and to create new matrices in bioremediation protocols.

\section{Beneficial effects of olive oil and olive by-products in}

human health: The Mediterranean diet, which is typical of Mediterranean countries, includes a diet rich in vegetables, legumes, fruits, fish, wine, and olive oil. This diet is associated with healthy aging, longevity, a low risk of incidence of age-related disorders such as cardiovascular diseases, hypertension, cancer, and chronic diseases (Figure 2) [32]. Interestingly, an abnormal production or degradation of reactive species can be considered the common denominator of all these diseases. The diet may represent a modifiable risk effector that can modulate several important cellular processes. Olive oil, a core of the Mediterranean diet, is an important lipid source having several beneficial effects on human health due to its specific chemical composition, which includes triacylglycerols, oleic acid, palmitic acid, linoleic acid, and minor compounds as tocopherols, terpenes, sterols, chlorophylls and phenolic molecules [33]. Over the last few years, particular attention has been paid to investigate the functional roles of the phenolic fraction for its antioxidant and anti-inflammatory activity [34]. Bioavailability studies in humans revealed that even the absorption of olive oil phenols is about $55-66 \%$, the final content of phenols may be too low to display beneficial responses [35]. Nevertheless, extra virgin olive oil (EVOO), which is 
considered a functional food, displayed beneficial effects on the digestive system being involved in the modulation of the arachidonic acid [36] and NF-kB signal transduction pathway [37]. Olive extracts, indeed, may modulate and contrast the inflammation by targeting TAK-1 mediated activation of the MAPK or by modulating the activities of immune system cells [38-39].

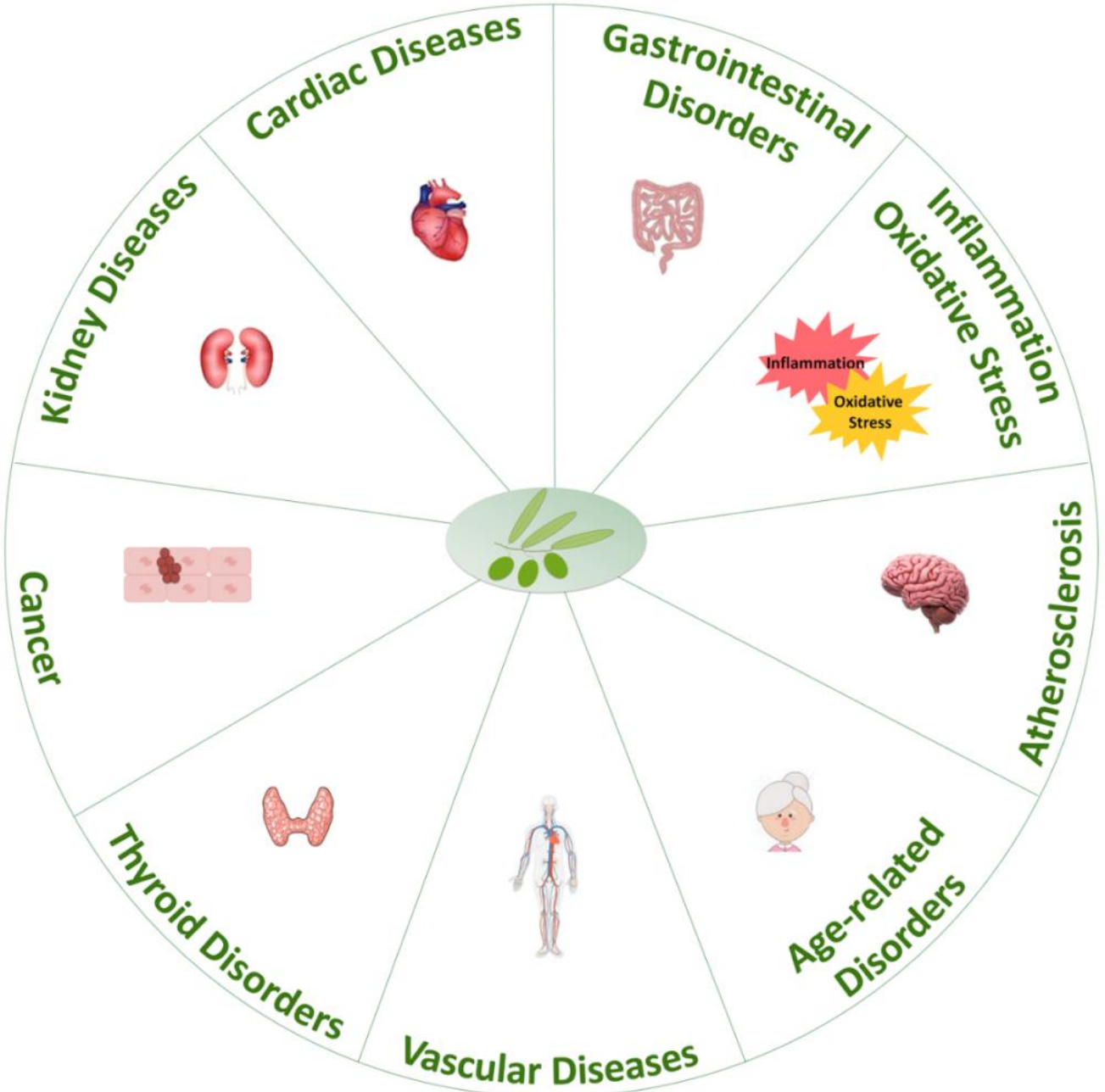

Figure 2. Diagram showing beneficial properties of olive oil and olive by-products on human health; in particular, on kidney diseases, cardiovascular diseases, gastrointestinal disorders, inflammation and oxidative stress, thyroid disorders, and cancer.

Beneficial actions on the gastrointestinal tract: The intestinal epithelium constitutes a physiological barrier involved in the reabsorption of nutrients and water as the intestines express several nutrient transporters [40]and aquaporins [41]. The physiology of the intestinal tract is tightly dependent on complex interplay between intrinsic and systemic factors modulating the immune response and the microbiota. Abnormal functioning of this complicated network may promote the onset of certain inflammatory disorders altering gastrointestinal functionality. It is well established that the Mediterranean diet, which includes consumption of fruits, vegetables, legumes, and olive oil, prevents several inflammatory diseases. In particular, EVOO and olive by-products contain an important fraction of polyphenols that possibly exert anti-inflammatory and antioxidant functions [42]. Importantly, while the plasma concentration of phenols might not be sufficient to promote significant systemic actions 
even after extensive intake, the concentration level in the lumen of the colon is incredibly high due to low reabsorption and bile secretion. Therefore, the colon represents an excellent choice organ to evaluate the physiological effects of bioactive compounds in EVOO and olive by-products [43]. In vitro studies showed that a phenolic fraction isolated from two monovarietal EVOOs prevented the oxidative stress induced by the tert-butyl hydroperoxide (tBHP) or diet oxysterols [44]. Following EVOO intake, hydroxytyrosol, tyrosol, oleuropein, and their metabolites are mainly identified in the lumen of the intestine where they might be committed to exert antioxidant and anti-inflammatory actions [45]. Extracts enriched in phenols and isolated from EVOO or olive by-products, including pomace and wastewater, modulated cell viability and counteracted the oxidative stress induced by tBHP in Caco-2 and HCT8 colon cell lines $[6 ; 46]$. Until now, pieces of evidence revealed that EVOO impaired antioxidant and antiinflammatory signals by inhibiting interleukin production and release and promoting the expression of anti-inflammatory genes. In Caco-2 cells, phenols derived from EVOO modulated the expression level of the inflammatory cytokine IL-8 possibly through p38 signaling [47]. In a mouse model of colitis, administration of EVOO reduced body weight and rectal bleeding possibly by reducing the expression level of pivotal cytokines involved in the onset of inflammation including IL-1 $\beta$, TGF $\beta$, IL-6 [37]. Interestingly, in healthy volunteers, intake of EVOO, enriched in polyphenols, improved insulin sensitivity and tightly regulated the expression level of miRNAs involved in metabolism, inflammation and cancer [48]. In patients with metabolic syndrome (MetS) and hepatic steatosis, EVOO administration for two months, significantly ameliorated crucial metabolic parameters and anthropometric data such as body weight, waist circumference, body mass index (BMI). This improvement was correlated with a reduction in alanine transaminase, IL-6, and IL-17A [49]. Moreover, phenols in EVOO have been suggested to be potentially useful in the management of obesity as they activated several intracellular pathways involving the stimulation of the AMP-activated protein kinase (AMPK) and sirtuin-1 that may promote the activation of the brown adipose tissue [50].

Effects of EVOO and olive by-products on the renal system: Renal disease represents a worldwide health issue due to its high clinical impact and costs. Renal tubular cells play key roles in water and solute reabsorption. This crucial resorptive function requires energy derived from mitochondria, which are also a natural source of reactive species. Therefore, the kidney is an organ particularly exposed to oxidative stress. Consistently, several renal disorders, including obstructive nephropathy, glomerular damage, polycystic kidney diseases, acute and chronic kidney injury, are associated with abnormal ROS signaling [10]. Several studies revealed that phenols in EVOO and olive by-products might be useful to counteract renal oxidative stress. Oleuropein, which is the main phenol in the OLE, displayed a beneficial effect in rats subjected for 3-days to unilateral ureteral obstruction (UUO) possibly by reducing oxidative stress and inflammation [51]. Moreover, hydroxytyrosol and tyrosol can be absorbed but also excreted in renal tubules where they might play a role as antioxidant and anti-inflammatory molecules. Interestingly, hydroxytyrosol and tyrosol reduced the $\mathrm{H}_{2} \mathrm{O}_{2}$ induced oxidative stress in proximal tubule LLC-PK cells [52], possibly by modulating the MAPK and PI3 kinase pathways [53-54]. Hydroxytyrosol, which is enriched in EVOO and olive wastewater, provided 
cytoprotection in three different renal cell models exposed to ochratoxin-A which is a contaminant mycotoxin isolated in foods [55]. Due to antiinflammatory actions, EVOO supplementation has been tested in patients with chronic kidney disease (CKD). CKD is a worldwide worrying health problem associated with impaired renal function caused by the loss of functional nephrons. Patients with CKD have a high risk of developing serious complications such as cardiovascular disease that increased mortality rates [56].The abnormal generation of ROS activated selective transcription factors that promoted the expression of pro-inflammatory genes causing renal injury [57]. In a pilot study, it has been found that consumption of EVOO (40 mL per day for 9 weeks), enriched in hydroxytyrosol, ameliorated the glomerular filtration rate, the lipid profile, the uric acid level and reduced the triglycerides concentration in CDK patients [33]. In vitro and in vivo findings from our group showed that exposure to OLE impaired the vasopressin-dependent AQP2 water reabsorption through the activation of the Calcium Sensing Receptor (CaSR) signaling. Specifically, we found that stimulation of the CaSR induced by OLE increased the AQP2 targeted miR-137 which is known to play a role in decreasing AQP2 expression and function [58]. In glioblastoma multiforme cells it has been demonstrated that OLE induced the expression of certain miRNAs such as miR-137 [59] that downregulated the Akt/mTOR pathway [60]. Exposure to oleuropein impaired Akt/mTOR signals by stimulating the calcium-induced CAMK and AMPK [61]. In renal proximal tubule cells, stimulation of the CaSR with the selective NPS-R568 agonist upregulated AMPK and decrease mTOR function [62]. Accordingly, exposure to OLE increased intracellular calcium level, reduced PKA activity and the cyst size in a 3D-cell culture model of autosomal dominant polycystic kidney disease [63]. Overall, these observations likely indicated that OLE may be administered as an adjuvant to treat disorders characterized by abnormal intracellular calcium concentration and associated with the downregulation of the CaSR signaling pathway.

EVOO and by-products on Cardiovascular health: The incidence risk of cardiovascular diseases (CVD) is increasing rapidly worldwide. Heart disorders are one of the main causes of death. Hypertension, abnormal plasmatic lipid profile, diabetes, and overweight can be considered the major risk factors for developing CVD. Numerous studies showed that cardiovascular dysfunctions are strictly related to uncontrolled oxidative stress and inflammation [64-65]. In this respect, a balanced diet enriched in antioxidant compounds may prevent the onset of the worsening of certain cardiovascular disturbs. Regular consumption of EVOO may provide benefit to human health due to the presence of several nutraceutical molecules exerting antioxidant and antiinflammatory functions, vasodilatory and antiplatelet aggregation [66]. EVOO intake, indeed, reduced the oxidation of $L D L$ that represents a priming event affecting endothelial cells and leading to arterial injury and the development of atherosclerosis [67]. In hypertensive patients, a single dose of virgin oil improved endothelial function by reducing the oxidation of LDL [68]. In traditional medicine, olive leaves were used as an antihypertensive remedy, possibly for their action on cardiomyocytes. In fact, in cardiomyocytes, exposure to OLE reduced the function of L-type calcium channels [69]. Initially, the beneficial effects of EVOO were mainly attributed to monounsaturated fatty acids (MUFA), such as oleic acid. Nevertheless, further studies revealed that a minor fraction containing 
antioxidant components plays a pivotal role for their nutraceutical properties. Hydroxytyrosol and oleuropein intake provided a protective effect against atherosclerosis due to a reduction of LDL lipid peroxidation [66;70]. Moreover, consumption of food enriched with $\mathrm{HT}$, isolated from olive wastewater, significantly decreased plasma thromboxane B2 generation, a known biomarker of platelet aggregation [71]. Administration of olive oil, with a different phenolic concentration, revealed that the intake of the oil containing the highest level of phenols is associated with a lower concentration of circulating oxidation markers [72]. In human vascular endothelial cells, oleuropein and HT decreased the LPS-induced expression of the vascular adhesion molecule-1 (VCAM-1), thereby impairing the cell adhesion of monocytes to endothelial cells [73]. At the molecular level, HT increased the expression of the antioxidant enzyme catalase through the AMPK pathway and reduced oxidative stress by stimulating the nuclear factor-E2-related factor-2 (Nrf2). Nrf2 is an important transcriptional factor that is stimulated in response to oxidative stress and promotes the expression of selective genes by interacting with the antioxidant response element (ARE) [74]. In endothelial cells treated with HT, Nrf2 modulates the PI3K/Akt and ERK1/2 signal pathways [75-76]. Moreover, in an animal model of myocardial injury induced by ischemia, perfusion with oleuropein before the ischemia reduced the abundances of creatine kinase and the oxidized glutathione which are specific biomarkers of cellular injury [77]. Also, in hypercholesterolemic rabbits subjected to ischemia/reperfusion, treatment with oleuropein significantly decreased the infarct size [78]. Together, these observations likely suggest that oleuropein may provide cardioprotection responses following coronary occlusion and may be beneficial for reperfused myocardium.

\section{Potential actions of olive oil and by-products in} cancer prevention: Cancer is the second leading worldwide cause of death following CVD. The most common cancers are from the lung, breast, colorectal, and pancreas, whose incidence has dramatically increased over the past few years. Cancer is a multifaceted disorder characterized by abnormal production of reactive species, inflammation, deregulated cell cycle, and angiogenesis [79]. It has been estimated that $30-50 \%$ of cancers may be prevented with a correct lifestyle and a healthy diet [80]. Several data proposed that consumption of olive oil may potentially reduce the risk of developing certain cancers. Epidemiological observations depicted a negative correlation between EVOO intake and the incidence to develop cancers [81]. Nevertheless, strong scientific evidence supporting this fascinating hypothesis is still missing. Several studies revealed, indeed, that bioactive compounds, such as monounsaturated fatty acid (MUFA) and oleic acid (OA), in EVOO play pivotal roles in contrasting tumorigenesis. In breast cancer cells, treatment with a physiological level of OA significantly reduced the expression of HER2 (which is a known oncogene playing a role in tumor progression) and the response to chemotherapy [82]. Lignans, secoiridoids, the phenolic compounds in oil and by-products may be also partially involved in the described anti-tumorigenic effects [83]. Consistently, it has been reported that bioactive compounds in oil and by-products may act as antioxidants [84-85], by modulating the immune system [86-87] and by controlling signals involved in cell proliferation and cell cycle [88-89]. In vivo and in vitro observations showed that EVOO may prevent the development of 
colon cancer [88;90-91]. In HT29 and Caco-2 colon cells olive oil supplementation reduces the expression of $\mathrm{COX}-2$ and $\mathrm{Bcl}-2$ [91]. The carcinogenic function of COX-2 is associated with the generation of prostaglandins that play an important role in promoting the survival and proliferation of colon cancer cells [92]. In HT29 cells, treatment with a dialdehydic form of decarboxy methyl ligstroside aglycone (p-HPEA-EDA), which is a phenolic substance in virgin oil, reduced cell proliferation and survival by downregulating COX-2 expression, AMPK signaling and the 12-0-tetradecanoylphorbol-13-acetate (TPA)-induced phosphorylation of extracellular signalregulated kinases 1/2 (ERK1/2)[93]. Hydroxytyrosol and oleuropein promote apoptosis in human melanoma cells and in human breast cancer cells by inducing an abnormal accumulation of ROS through the inhibition of the NF-kB factor [94-95]. Other EVOO components, including oleanolic acid, oleocanthal, and pinoresinol displayed significant antiproliferative effects in breast cancer cells. Oleocanthal also blocked cell invasion [95]. Oleacein, the most abundant secoiridoids in EVOO, has a strong antiproliferative action in SH-SY5Y human neuroblastoma cells. In particular, it has been demonstrated that oleacein blocks cell cycle in the $S$ phase and stimulates the apoptotic cell program through the activation of Bax and p53, the downregulation of $\mathrm{Bcl}-2$ and the phosphorylation of the transcription factor STAT3 [96].

\section{CONCLUSION}

Emerging evidence in biological and clinical fields revealed and proposed the potential health benefits of EVOO and olive by-products to prevent and improve certain chronic disturbances, including those affecting gastrointestinal function, renal physiology, and cardiovascular diseases. Interestingly, new data are now available showing the effect of specific phenols in olive oil and by-products to counteract tumorigenesis at different molecular levels. Novel and alternative use of bioactive compounds in wastes and by-products as a food preservative are emerging to generate green biofuel or to produce specific matrices in bioremediation plans. More focused research is needed to clarify the potential role and application of bioactive compounds having healthy effects and application in industry processes.

Abbreviations: EVOO: extra virgin olive oil, OP: olive pomace, OWW: olive wastewater, OLE: olive leaf extracts, HT: hydroxytyrosol, RS: reactive species, ROS: Reactive Oxygen Species, MetS: metabolic syndrome, CVD: cardiovascular diseases, CKD: chronic kidney disease

Author's Contributions: G. Tamma conceived of and designed the review. M. Centrone, M. Ranieri and G. Tamma participated in drafting the manuscript. A. Di Mise, M. D'Agostino, M. Venneri and G. Valenti reviewed the manuscript, and all authors were engaged in the manuscript work. All authors have read and approved the final manuscript.

Competing Interests: None of the authors have competing interests to declare.

Acknowledgements and Funding: M.R. is supported by POR Puglia 2014/2020 - Asse X - Azione 10.4. Research for Innovation - REFIN (Code n. 4FC8E072); A.D.M. is supported by "Attrazione e Mobilità dei Ricercatori, PON “R\&I” 2014-2020, Azione I.2" (code AIM1893457-3, linea 1).

\section{REFERENCES}

1. Emma MR, Augello G, Di Stefano V, Azzolina A, Giannitrapani L, Montalto G, Cervello M, Cusimano A. Potential Uses of Olive Oil Secoiridoids for the Prevention and Treatment of Cancer: A Narrative Review of Preclinical 
Studies. International journal of molecular sciences 2021, 22. doi:10.3390/ijms22031234.

2. Difonzo G, Russo A, Trani A, Paradiso VM, Ranieri M, Pasqualone A, Summo C, Tamma G, Silletti R, Caponio F. Green extracts from Coratina olive cultivar leaves: Antioxidant characterization and biological activity. Journal of Functional Foods 2017, 31, 63-70. doi:http://dx.doi.org/10.1016/j.jff.2017.01.039.

3. Lee OH, Lee BY, Lee J, Lee HB, Son JY, Park CS, Shetty K, Kim YC. Assessment of phenolics-enriched extract and fractions of olive leaves and their antioxidant activities. Bioresour Technol 2009, 100, 6107-6113. doi:10.1016/j.biortech.2009.06.059.

4. Silva AFR, Resende D, Monteiro M, Coimbra MA, Silva AMS, Cardoso SM. Application of Hydroxytyrosol in the Functional Foods Field: From Ingredient to Dietary Supplements. Antioxidants (Basel) 2020, 9. doi:10.3390/antiox9121246.

5. Ranieri M, Di Mise A, Difonzo G, Centrone M, Venneri M, Pellegrino T, Russo A, Mastrodonato M, Caponio F, Valenti G, Tamma G. Green olive leaf extract (OLE) provides cytoprotection in renal cells exposed to low doses of cadmium. PloS one 2019, 14, e0214159. doi:10.1371/journal.pone.0214159.

6. Centrone M, D'Agostino M, Difonzo G, De Bruno A, Di Mise A, Ranieri M, Montemurro C, Valenti G, Poiana M, Caponio F, Tamma G. Antioxidant Efficacy of Olive By-Product Extracts in Human Colon HCT8 Cells. Foods (Basel, Switzerland) 2020, 10. doi:10.3390/foods10010011.

7. Hassen I, Casabianca H, Hosni K. Biological activities of the natural antioxidant oleuropein: Exceeding the expectation - A mini-review. Journal of Functional Foods 2015, 18, 926940. doi:https://doi.org/10.1016/j.jff.2014.09.001.

8. Goto S, Nakamura A. Age-associated, oxidatively modified proteins: A critical evaluation. Age (Omaha) 1997, 20, 8189. doi:10.1007/s11357-997-0008-y.

9. Tamma G, Valenti G, Grossini E, Donnini S, Marino A, Marinelli RA, Calamita G. Aquaporin Membrane Channels in Oxidative Stress, Cell Signaling, and Aging: Recent Advances and Research Trends. Oxid Med Cell Longev 2018, 2018, 1501847. doi:10.1155/2018/1501847.

10. Tamma G, Valenti G. Evaluating the Oxidative Stress in Renal Diseases: What Is the Role for S-Glutathionylation? Antioxid Redox Signal 2016, 25, 147-164. doi:10.1089/ars.2016.6656.

11. Oi-Kano Y, Kawada T, Watanabe T, Koyama F, Watanabe $K_{\text {, }}$ Senbongi R, Iwai K. Oleuropein supplementation increases urinary noradrenaline and testicular testosterone levels and decreases plasma corticosterone level in rats fed high- protein diet. The Journal of nutritional biochemistry 2013, 24, 887-893. doi:10.1016/j.jnutbio.2012.06.003.

12. Pang KL, Lumintang JN, Chin KY. Thyroid-Modulating Activities of Olive and Its Polyphenols: A Systematic Review. Nutrients 2021, 13. doi:10.3390/nu13020529.

13. Farooq $M$, Ali $S$, Zubair $M$, Ullah $Q$, Jamil $H$, Haroon $M$, Ghaffar A. Effect of feed supplementation with olive oil on serum testosterone, triiodothyronine, thyroxine and some biochemical metabolites in teddy goat bucks. Asian J Agric Biol 2019, 7, 116-121.

14. Al-Qarawi AA, Al-Damegh MA, ElMougy SA. Effect of freeze dried extract of Olea europaea on the pituitary-thyroid axis in rats. Phytotherapy research: PTR 2002, 16, 286-287. doi:10.1002/ptr.855.

15. Mekircha F, Chebab S, Gabbianelli R, Leghouchi E. The possible ameliorative effect of Olea europaea $L$. oil against deltamethrin-induced oxidative stress and alterations of serum concentrations of thyroid and reproductive hormones in adult female rats. Ecotoxicology and environmental safety 2018, 161, 374-382. doi:10.1016/j.ecoenv.2018.05.086.

16. Rodrigues F, Pimentel FB, Oliveira MBPP. Olive byproducts: Challenge application in cosmetic industry. Industrial Crops and Products 2015, 70, 116-124. doi:https://doi.org/10.1016/j.indcrop.2015.03.027.

17. Berbel J, Posadillo A. Review and Analysis of Alternatives for the Valorisation of Agro-Industrial Olive Oil ByProducts. Sustainability 2018, 10, 1-9. doi:10.3390/su10010237.

18. Koutrotsios G, Larou E, Mountzouris KC, Zervakis GI. Detoxification of Olive Mill Wastewater and Bioconversion of Olive Crop Residues into High-Value-Added Biomass by the Choice Edible Mushroom Hericium erinaceus. Appl Biochem Biotechnol 2016, 180, 195-209. doi:10.1007/s12010-016-2093-9.

19. Rupic V, Bozikov V, Bozac R, Muzic S, Vranesic N, Dikic M. Effect of feeding olive by-products on certain blood parameters and serum enzyme activities of fattening rabbits. Acta Vet Hung 1999, 47, 65-75. doi:10.1556/AVet.47.1999.1.6.

20. Rupic V, Jerkovic I, Bozac R, Glowattzky D, Muzic S, Hrabak V. Olive by-products in pig fattening. Acta Vet Hung 1997, 45, 53-66.

21. Branciari R, Galarini R, Miraglia D, Ranucci D, Valiani A, Giusepponi D, Servili M, Acuti G, Pauselli M, TrabalzaMarinucci M. Dietary Supplementation with Olive Mill Wastewater in Dairy Sheep: Evaluation of Cheese Characteristics and Presence of Bioactive Molecules. Animals (Basel) 2020, 10. doi:10.3390/ani10111941. 
22. Garcia AV, Alvarez-Perez OB, Rojas R, Aguilar CN, Garrigos MC. Impact of Olive Extract Addition on Corn Starch-Based Active Edible Films Properties for Food Packaging Applications. Foods (Basel, Switzerland) 2020, 9. doi:10.3390/foods9091339.

23. Gullon P, Gullon B, Astray G, Carpena M, Fraga-Corral M, Prieto MA, Simal-Gandara J. Valorization of by-products from olive oil industry and added-value applications for innovative functional foods. Food Res Int 2020, 137, 109683. doi:10.1016/j.foodres.2020.109683.

24. Anastopoulos I, Massas I, Ehaliotis C. Use of residues and by-products of the olive-oil production chain for the removal of pollutants from environmental media: A review of batch biosorption approaches. J Environ Sci Health A Tox Hazard Subst Environ Eng 2015, 50, 677-718. doi:10.1080/10934529.2015.1011964.

25. Mahmoudi A, Ghorbel H, Feki I, Bouallagui Z, Guermazi F, Ayadi L, Sayadi S. Oleuropein and hydroxytyrosol protect rats' pups against bisphenol A induced hypothyroidism. Biomedicine and pharmacotherapy 2018, 103, 1115-1126. doi:10.1016/j.biopha.2018.03.004.

26. Takeda $Y$, Jamsransuren $D$, Matsuda $S$, Crea R, Ogawa $H$. The SARS-CoV-2-Inactivating Activity of HydroxytyrosolRich Aqueous Olive Pulp Extract (HIDROX $\left.\left({ }^{\circledR}\right)\right)$ and Its Use as a Virucidal Cream for Topical Application. Viruses 2021, 13. doi:10.3390/v13020232.

27. Ünlü $A E$. Green and Non-conventional Extraction of Bioactive Compounds from Olive Leaves: Screening of Novel Natural Deep Eutectic Solvents and Investigation of Process Parameters. Waste and biomass valorization 2021, 10.1007/s12649-021-01411-3, 1-18. doi:10.1007/s12649021-01411-3.

28. Theoharides TC, Cholevas C, Polyzoidis K, Politis A. LongCOVID syndrome-associated brain fog and chemofog: Luteolin to the rescue. BioFactors (Oxford, England) 2021, 47, 232-241. doi:10.1002/biof.1726.

29. Cedola A, Palermo C, Centonze D, Del Nobile MA, Conte A. Characterization and Bio-Accessibility Evaluation of Olive Leaf Extract-Enriched "Taralli". Foods (Basel, Switzerland) 2020, 9. doi:10.3390/foods9091268.

30. Difonzo G, Pasqualone A, Silletti R, Cosmai L, Summo C, Paradiso VM, Caponio F. Use of olive leaf extract to reduce lipid oxidation of baked snacks. Food Res Int 2018, 108, 4856. doi:10.1016/j.foodres.2018.03.034.

31. Panza O, Lacivita V, Palermo C, Conte A, Del Nobile MA. Food By-Products to Extend Shelf Life: The Case of Cod Sticks Breaded with Dried Olive Paste. Foods (Basel, Switzerland) 2020, 9. doi:10.3390/foods9121902.
32. Tripoli E, Giammanco M, Tabacchi G, Di Majo D, Giammanco S, La Guardia M. The phenolic compounds of olive oil: structure, biological activity and beneficial effects on human health. Nutr Res Rev 2005, 18, 98-112. doi:10.1079/NRR200495.

33. Romani A, Bernini R, Noce A, Urciuoli S, Di Lauro M, Pietroboni Zaitseva A, Marrone G, Di Daniele N. Potential Beneficial Effects of Extra Virgin Olive Oils Characterized by High Content in Minor Polar Compounds in Nephropathic Patients: A Pilot Study. Molecules (Basel, Switzerland) 2020, 25. doi:10.3390/molecules25204757.

34. Romani A, leri F, Urciuoli S, Noce A, Marrone G, Nediani C, Bernini R. Health Effects of Phenolic Compounds Found in Extra-Virgin Olive Oil, By-Products, and Leaf of Olea europaea L. Nutrients 2019, 11. doi:10.3390/nu11081776.

35. Vissers MN, Zock PL, Katan MB. Bioavailability and antioxidant effects of olive oil phenols in humans: a review. Eur J Clin Nutr 2004, 58, 955-965. doi:10.1038/sj.ejcn.1601917.

36. Llorente-Cortés V, Estruch R, Mena MP, Ros E, González MA, Fitó M, Lamuela-Raventós RM, Badimon L. Effect of Mediterranean diet on the expression of pro-atherogenic genes in a population at high cardiovascular risk. Atherosclerosis 2010, 208, 442-450. doi:10.1016/j.atherosclerosis.2009.08.004.

37. Cariello M, Contursi A, Gadaleta RM, Piccinin E, De Santis S, Piglionica M, Spaziante AF, Sabbà C, Villani G, Moschetta A. Extra-Virgin Olive Oil from Apulian Cultivars and Intestinal Inflammation. Nutrients 2020, 12. doi:10.3390/nu12041084.

38. De Cicco P, Maisto M, Tenore GC, lanaro A. Olive Leaf Extract, from Olea europaea L., Reduces Palmitate-Induced Inflammation via Regulation of Murine Macrophages Polarization. Nutrients 2020, 12. doi:10.3390/nu12123663.

39. Song C, Kim MY, Cho JY. Olea europaea Suppresses Inflammation by Targeting TAK1-Mediated MAP Kinase Activation. Molecules (Basel, Switzerland) 2021, 26. doi:10.3390/molecules26061540.

40. Kiela PR, Ghishan FK. Physiology of Intestinal Absorption and Secretion. Best practice and research. Clinical gastroenterology 2016, 30, 145-159. doi:10.1016/j.bpg.2016.02.007.

41. Zhu C, Chen Z, Jiang Z. Expression, Distribution and Role of Aquaporin Water Channels in Human and Animal Stomach and Intestines. International journal of molecular sciences 2016, 17. doi:10.3390/ijms17091399.

42. Cicerale S, Lucas L, Keast RS. Antimicrobial, antioxidant and anti-inflammatory phenolic activities in extra virgin 
olive oil. Current opinion in biotechnology 2012, 23, 129 135. doi:10.1016/j.copbio.2011.09.006

43. Halliwell B, Rafter J, Jenner A. Health promotion by flavonoids, tocopherols, tocotrienols, and other phenols: direct or indirect effects? Antioxidant or not? Am J Clin Nutr 2005, 81, 268S-276S. doi:10.1093/ajen/81.1.268S.

44. Incani A, Serra G, Atzeri A, Melis MP, Serreli G, Bandino G, Sedda $\mathrm{P}$, Campus $\mathrm{M}$, Tuberoso $\mathrm{Cl}$, Deiana $\mathrm{M}$. Extra virgin olive oil phenolic extracts counteract the pro-oxidant effect of dietary oxidized lipids in human intestinal cells. Food Chem Toxicol 2016, 90, 171-180. doi:10.1016/j.fct.2016.02.015.

45. Corona G, Spencer JP, Dessì MA. Extra virgin olive oil phenolics: absorption, metabolism, and biological activities in the Gl tract. Toxicology and industrial health 2009, 25, 285-293. doi:10.1177/0748233709102951.

46. Atzeri A, Lucas R, Incani A, Penalver P, Zafra-Gomez A, Melis MP, Pizzala R, Morales JC, Deiana M. Hydroxytyrosol and tyrosol sulfate metabolites protect against the oxidized cholesterol pro-oxidant effect in Caco-2 human enterocyte-like cells. Food Funct 2016, 7, 337-346. doi:10.1039/c5fo00074b.

47. Muto E, Dell'Agli M, Sangiovanni E, Mitro N, Fumagalli M, Crestani M, De Fabiani E, Caruso D. Olive oil phenolic extract regulates interleukin-8 expression by transcriptional and posttranscriptional mechanisms in Caco-2 cells. Mol Nutr Food Res 2015, 59, 1217-1221. doi:10.1002/mnfr.201400800.

48. D'Amore S, Vacca M, Cariello M, Graziano G, D'Orazio A, Salvia R, Sasso RC, Sabba C, Palasciano G, Moschetta A. Genes and miRNA expression signatures in peripheral blood mononuclear cells in healthy subjects and patients with metabolic syndrome after acute intake of extra virgin olive oil. Biochim Biophys Acta 2016, 1861, 1671-1680. doi:10.1016/j.bbalip.2016.07.003.

49. Patti AM, Carruba G, Cicero AFG, Banach M, Nikolic D, Giglio RV, Terranova A, Soresi M, Giannitrapani L, Montalto G, Stoian AP, Banerjee Y, Rizvi AA, Toth PP, Rizzo M. Daily Use of Extra Virgin Olive Oil with High Oleocanthal Concentration Reduced Body Weight, Waist Circumference, Alanine Transaminase, Inflammatory Cytokines and Hepatic Steatosis in Subjects with the Metabolic Syndrome: A 2-Month Intervention Study. Metabolites 2020, 10. doi:10.3390/metabo10100392.

50. Melguizo Rodríguez L, Illescas-Montes R, Costela-Ruiz VJ, García-Martínez O. Stimulation of brown adipose tissue by polyphenols in extra virgin olive oil. Critical reviews in food science and nutrition 2020,
10.1080/10408398.2020.1799930,

$1-8$. doi:10.1080/10408398.2020.1799930.

51. Kaeidi A, Sahamsizadeh A, Allahtavakoli M, Fatemi I, Rahmani M, Hakimizadeh E, Hassanshahi J. The effect of oleuropein on unilateral ureteral obstruction inducedkidney injury in rats: the role of oxidative stress, inflammation and apoptosis. Mol Biol Rep 2020, 47, 13711379. doi:10.1007/s11033-019-05237-0.

52. Loru D, Incani A, Deiana M, Corona G, Atzeri A, Melis MP, Rosa A, Dessi MA. Protective effect of hydroxytyrosol and tyrosol against oxidative stress in kidney cells. Toxicology and industrial health 2009, 25, 301-310. doi:10.1177/0748233709103028.

53. Deiana M, Incani A, Rosa A, Atzeri A, Loru D, Cabboi B, Paola Melis M, Lucas R, Morales JC, Assunta Dessi M. Hydroxytyrosol glucuronides protect renal tubular epithelial cells against $\mathrm{H}(2) \mathrm{O}(2)$ induced oxidative damage. Chem Biol Interact 2011, 193, 232-239. doi:10.1016/j.cbi.2011.07.002.

54. Incani A, Deiana M, Corona G, Vafeiadou K, Vauzour D, Dessi MA, Spencer JP. Involvement of ERK, Akt and JNK signalling in $\mathrm{H} 2 \mathrm{O} 2$-induced cell injury and protection by hydroxytyrosol and its metabolite homovanillic alcohol. Mol Nutr Food Res 2010, 54, 788-796. doi:10.1002/mnfr.200900098.

55. Crupi R, Palma E, Siracusa R, Fusco R, Gugliandolo E, Cordaro M, Impellizzeri D, De Caro C, Calzetta L, Cuzzocrea S, Di Paola R. Protective Effect of Hydroxytyrosol Against Oxidative Stress Induced by the Ochratoxin in Kidney Cells: in vitro and in vivo Study. Front Vet Sci 2020, 7, 136. doi:10.3389/fvets.2020.00136.

56. Romagnani P, Remuzzi G, Glassock R, Levin A, Jager KJ, Tonelli M, Massy Z, Wanner C, Anders HJ. Chronic kidney disease. Nat Rev Dis Primers 2017, 3, 17088. doi:10.1038/nrdp.2017.88

57. Podkowinska A, Formanowicz D. Chronic Kidney Disease as Oxidative Stress- and Inflammatory-Mediated Cardiovascular Disease. Antioxidants (Basel) 2020, 9. doi:10.3390/antiox9080752.

58. Ranieri M, Di Mise A, Centrone M, D'Agostino M, Tingskov SJ, Venneri M, Pellegrino T, Difonzo G, Caponio F, Norregaard R, Valenti G, Tamma G. Olive Leaf Extract (OLE) impaired vasopressin-induced aquaporin-2 trafficking through the activation of the calcium-sensing receptor. Scientific reports 2021, 11, 4537. doi:10.1038/s41598-02183850-5.

59. Tezcan G, Tunca B, Bekar A, Budak F, Sahin S, Cecener G, Egeli U, Taskapilioglu MO, Kocaeli H, Tolunay S, Malyer H, Demir C, Tumen G. Olea europaea leaf extract improves 
the treatment response of GBM stem cells by modulating miRNA expression. Am J Cancer Res 2014, 4, 572-590.

60. Wang L, Liu J, Zhong Z, Gong X, Liu W, Shi L, Li X. PTP4A3 is a target for inhibition of cell proliferatin, migration and invasion through $\mathrm{Akt} / \mathrm{mTOR}$ signaling pathway in glioblastoma under the regulation of miR-137. Brain Res 2016, 1646, 441-450. doi:10.1016/j.brainres.2016.06.026.

61. Rigacci S, Miceli C, Nediani C, Berti A, Cascella R, Pantano D, Nardiello P, Luccarini I, Casamenti F, Stefani M. Oleuropein aglycone induces autophagy via the AMPK/mTOR signalling pathway: a mechanistic insight. Oncotarget 2015, 6, 35344-35357. doi:10.18632/oncotarget.6119.

62. Di Mise A, Tamma G, Ranieri M, Centrone M, van den Heuvel L, Mekahli D, Levtchenko EN, Valenti G. Activation of Calcium-Sensing Receptor increases intracellular calcium and decreases CAMP and mTOR in PKD1 deficient cells. Scientific reports 2018, 8, 5704. doi:10.1038/s41598018-23732-5.

63. Toteda G, Vizza D, Lupinacci S, Perri A, Scalise MF, Indiveri C, Puoci F, Parisi OI, Lofaro D, La Russa A, Gigliotti P, Leone F, Pochini L, Bonofiglio R. Olive leaf extract counteracts cell proliferation and cyst growth in an in vitro model of autosomal dominant polycystic kidney disease. Food Funct 2018, 9, 5925-5935. doi:10.1039/c8fo01481g.

64. Jin R, Yang G, Li G. Inflammatory mechanisms in ischemic stroke: role of inflammatory cells. J Leukoc Biol 2010, 87, 779-789. doi:10.1189/jlb.1109766.

65. Yu Y, Sun $G$, Luo Y, Wang M, Chen R, Zhang J, Ai Q, Xing N, Sun $\mathrm{X}$. Cardioprotective effects of Notoginsenoside R1 against ischemia/reperfusion injuries by regulating oxidative stress- and endoplasmic reticulum stress- related signaling pathways. Scientific reports 2016, 6, 21730. doi:10.1038/srep21730.

66. Bulotta S, Celano M, Lepore SM, Montalcini T, Pujia A, Russo D. Beneficial effects of the olive oil phenolic components oleuropein and hydroxytyrosol: focus on protection against cardiovascular and metabolic diseases. J TransI Med 2014, 12, 219. doi:10.1186/s12967-014-02199.

67. Berrougui $H$, Ikhlef S, Khalil A. Extra Virgin Olive Oil Polyphenols Promote Cholesterol Efflux and Improve HDL Functionality. Evid Based Complement Alternat Med 2015, 2015, 208062. doi:10.1155/2015/208062.

68. Valls RM, Farras M, Suarez M, Fernandez-Castillejo $S$, Fito M, Konstantinidou V, Fuentes F, Lopez-Miranda J, Giralt M, Covas MI, Motilva MJ, Solà R. Effects of functional olive oil enriched with its own phenolic compounds on endothelial function in hypertensive patients. A randomised controlled trial. Food Chem 2015, 167, 30-35. doi:10.1016/j.foodchem.2014.06.107.

69. Scheffler A, Rauwald HW, Kampa B, Mann U, Mohr FW, Dhein $\mathrm{S}$. Olea europaea leaf extract exerts L-type $\mathrm{Ca}(2+)$ channel antagonistic effects. J Ethnopharmacol 2008, 120. 233-240, doi:10.1016/j.jep.2008.08.018.

70. Carr AC, Tijerina T, Frei B. Vitamin C protects against and reverses specific hypochlorous acid- and chloraminedependent modifications of low-density lipoprotein. Biochem J 2000, 346 Pt 2, 491-499.

71. Leger CL, Carbonneau MA, Michel F, Mas E, Monnier L, Cristol JP, Descomps B. A thromboxane effect of a hydroxytyrosol-rich olive oil wastewater extract in patients with uncomplicated type I diabetes. Eur I Clin Nutr 2005, 59, 727-730. doi:10.1038/sj.ejcn.1602133.

72. de la Torre-Carbot K, Chavez-Servin JL, Jauregui O, Castellote Al, Lamuela-Raventos RM, Nurmi T, Poulsen $\mathrm{HE}$, Gaddi AV, Kaikkonen J, Zunft HF, Kiesewetter H, Fitó M, Covas MI, López-Sabater MC. Elevated circulating LDL phenol levels in men who consumed virgin rather than refined olive oil are associated with less oxidation of plasma LDL. J Nutr 2010, 140, 501-508. doi:10.3945/jn.109.112912.

73. Carluccio MA, Siculella L, Ancora MA, Massaro M, Scoditti E, Storelli C, Visioli F, Distante A, De Caterina R. Olive oil and red wine antioxidant polyphenols inhibit endothelial activation: antiatherogenic properties of Mediterranean diet phytochemicals. Arterioscler Thromb Vasc Biol 2003, 23, 622-629. doi:10.1161/01.ATV.0000062884.69432.A0.

74. Hilliard A, Mendonca P, Russell TD, Soliman KFA. The Protective Effects of Flavonoids in Cataract Formation through the Activation of Nrf2 and the Inhibition of MMP9. Nutrients 2020, 12. doi:10.3390/nu12123651.

75. Martin MA, Ramos S, Granado-Serrano AB, RodriguezRamiro I, Trujillo M, Bravo L, Goya L. Hydroxytyrosol induces antioxidant/detoxificant enzymes and Nrf2 translocation via extracellular regulated kinases and phosphatidylinositol-3-kinase/protein kinase B pathways in HepG2 cells. Mol Nutr Food Res 2010, 54, 956-966. doi:10.1002/mnfr.200900159.

76. Zrelli $\mathrm{H}$, Matsuoka M, Kitazaki S, Araki M, Kusunoki M, Zarrouk M, Miyazaki H. Hydroxytyrosol induces proliferation and cytoprotection against oxidative injury in vascular endothelial cells: role of Nrf2 activation and HO-1 induction. J Agric Food Chem 2011, 59, 4473-4482. doi:10.1021/jf104151d.

77. Manna C, Migliardi V, Golino P, Scognamiglio A, Galletti P, Chiariello M, Zappia V. Oleuropein prevents oxidative myocardial injury induced by ischemia and reperfusion. 
The Journal of nutritional biochemistry 2004, 15, 461-466. doi:10.1016/j.jnutbio.2003.12.010.

78. Andreadou I, lliodromitis EK, Mikros E, Constantinou M, Agalias A, Magiatis P, Skaltsounis AL, Kamber E, TsantiliKakoulidou A, Kremastinos DT. The olive constituent oleuropein exhibits anti-ischemic, antioxidative, and hypolipidemic effects in anesthetized rabbits. J Nutr 2006, 136, 2213-2219. doi:10.1093/jn/136.8.2213.

79. Byers T. What can randomized controlled trials tell us about nutrition and cancer prevention? CA Cancer J Clin 1999, 49, 353-361. doi:10.3322/canjclin.49.6.353.

80. Anand $P$, Kunnumakkara $A B$, Sundaram $C$, Harikumar KB, Tharakan ST, Lai OS, Sung B, Aggarwal BB. Cancer is a preventable disease that requires major lifestyle changes. Pharm Res 2008, 25, 2097-2116. doi:10.1007/s11095-0089661-9.

81. Farras M, Almanza-Aguilera E, Hernaez A, Agusti N, Julve J, Fito $M$, Castaner O. Beneficial effects of olive oil and Mediterranean diet on cancer physio-pathology and incidence. Semin Cancer Biol 2020. doi:10.1016/j.semcancer.2020.11.011.

82. Colomer R, Menendez JA. Mediterranean diet, olive oil and cancer. Clin Transl Oncol 2006, 8, 15-21. doi:10.1007/s12094-006-0090-0.

83. Owen RW, Giacosa A, Hull WE, Haubner R, Spiegelhalder B, Bartsch $\mathrm{H}$. The antioxidant/anticancer potential of phenolic compounds isolated from olive oil. Eur J Cancer 2000, 36, 1235-1247. doi:10.1016/s0959-8049(00)001039.

84. Duthie SJ, Dobson VL. Dietary flavonoids protect human colonocyte DNA from oxidative attack in vitro. Eur J Nutr 1999, 38, 28-34. doi:10.1007/s003940050043.

85. Sun L, Luo C, Liu J. Hydroxytyrosol induces apoptosis in human colon cancer cells through ROS generation. Food Funct 2014, 5, 1909-1914. doi:10.1039/c4fo00187g.

86. Magrone $T$, Spagnoletta A, Salvatore $R$, Magrone $M$, Dentamaro F, Russo MA, Difonzo G, Summo C, Caponio F, Jirillo E. Olive Leaf Extracts Act as Modulators of the Human Immune Response. Endocr Metab Immune Disord Drug Targets 2018, 18, 8593.doi:10.2174/1871530317666171116110537.

87. Safonova GM, Shilov $Y$, Perevozchikov AB. Protective effects of plant polyphenols on the immune system in acute stress. Dokl Biol Sci 2001, 378, 233-235. doi:10.1023/a:1019210607120.
88. Corona G, Deiana M, Incani A, Vauzour D, Dessi MA, Spencer JP. Inhibition of p38/CREB phosphorylation and COX-2 expression by olive oil polyphenols underlies their anti-proliferative effects. Biochem Biophys Res Commun 2007, 362, 606-611. doi:10.1016/j.bbrc.2007.08.049.

89. Plaumann B, Fritsche M, Rimpler H, Brandner G, Hess RD. Flavonoids activate wild-type p53. Oncogene 1996, 13, 1605-1614.

90. Di Francesco A, Falconi A, Di Germanio C, Micioni Di Bonaventura MV, Costa A, Caramuta S, Del Carlo M, Compagnone D, Dainese E, Cifani C, Maccarrone M, D'Addario C. Extravirgin olive oil up-regulates $\mathrm{CB}(1)$ tumor suppressor gene in human colon cancer cells and in rat colon via epigenetic mechanisms. The Journal of nutritional biochemistry 2015, 26, 250-258. doi:10.1016/j.jnutbio.2014.10.013.

91. Llor X, Pons E, Roca A, Alvarez M, Mane J, FernandezBanares F, Gassull MA. The effects of fish oil, olive oil, oleic acid and linoleic acid on colorectal neoplastic processes. Clin Nutr 2003, 22, 71-79. doi:10.1054/clnu.2002.0627.

92. Karpisheh V, Joshi N, Zekiy AO, Beyzai B, Hojjat-Farsangi M, Namdar A, Edalati M, Jadidi-Niaragh F. EP4 receptor as a novel promising therapeutic target in colon cancer. Pathol Res Pract 2020, 216, 153247. doi:10.1016/j.prp.2020.153247.

93. Khanal P, Oh WK, Yun HJ, Namgoong GM, Ahn SG, Kwon SM, Choi HK, Choi HS. p-HPEA-EDA, a phenolic compound of virgin olive oil, activates AMP-activated protein kinase to inhibit carcinogenesis. Carcinogenesis 2011, 32, 545553. doi:10.1093/carcin/bgr001.

94. Costantini F, Di Sano C, Barbieri G. The Hydroxytyrosol Induces the Death for Apoptosis of Human Melanoma Cells. International journal of molecular sciences 2020, 21. doi:10.3390/ijms21218074.

95. Donovan MG, Selmin OI, Stillwater BJ, Neumayer LA, Romagnolo DF. Do Olive and Fish Oils of the Mediterranean Diet Have a Role in Triple Negative Breast Cancer Prevention and Therapy? An Exploration of Evidence in Cells and Animal Models. Front Nutr 2020, 7, 571455 doi:10.3389/fnut.2020.571455.

96. Cirmi S, Celano M, Lombardo GE, Maggisano V, Procopio A, Russo D, Navarra M. Oleacein inhibits STAT3, activates the apoptotic machinery, and exerts anti-metastatic effects in the SH-SY5Y human neuroblastoma cells. Food Funct 2020, 11, 3271-3279. doi:10.1039/d0fo00089b. 